\title{
Thermospheric winds and temperatures above Mawson, Antarctica, observed with an all-sky imaging, Fabry-Perot spectrometer
}

\author{
C. Anderson ${ }^{1}$, M. Conde ${ }^{2}$, P. Dyson ${ }^{1}$, T. Davies ${ }^{1}$, and M. J. Kosch ${ }^{3}$ \\ ${ }^{1}$ Department of Physics, La Trobe University, Australia \\ ${ }^{2}$ Geophysical Institute, University of Alaska, USA \\ ${ }^{3}$ Department of Communication Systems, Lancaster University, UK
}

Received: 7 November 2008 - Revised: 3 April 2009 - Accepted: 6 May 2009 - Published: 15 May 2009

\begin{abstract}
A new all-sky imaging Fabry-Perot spectrometer has been installed at Mawson station $\left(67^{\circ} 36^{\prime} \mathrm{S}, 62^{\circ} 52^{\prime} \mathrm{E}\right)$, Antarctica. This instrument is capable of recording independent spectra from many tens of locations across the sky simultaneously. Useful operation began in March 2007, with spectra recorded on a total of 186 nights. Initial analysis has focused on the large-scale daily and average behavior of winds and temperatures derived from observations of the $630.0 \mathrm{~nm}$ airglow line of atomic oxygen, originating from a broad layer centered around $240 \mathrm{~km}$ altitude, in the ionospheric F-region.
\end{abstract}

The 1993 Horizontal Wind Model (HWM93), NRLMSISE-00 atmospheric model, and the Coupled Thermosphere/Ionosphere Plasmasphere (CTIP) model were used for comparison. During the geomagnetically quiet period studied, observed winds and temperatures were generally well modelled, although temperatures were consistently higher than NRLMSISE-00 predicted, by up to $100 \mathrm{~K}$. CTIP temperatures better matched our data, particularly later in the night, but predicted zonal winds which were offset from those observed by $70-180 \mathrm{~ms}^{-1}$ westward. During periods of increased activity both winds and temperatures showed much greater variability over time-scales of less than an hour. For the active night presented here, a period of $45 \mathrm{~min}$ saw wind speeds decrease by around $180 \mathrm{~ms}^{-1}$, and temperatures increase by approximately $100 \mathrm{~K}$. Activeperiod winds were poorly modelled by HWM93 and CTIP, although observed median temperatures were in better agreement with NRLMSISE-00 during such periods.

Average behavior was found to be generally consistent with previous studies of thermospheric winds above Mawson. The collected data set was representative of quiet geomagnetic and solar conditions. Geographic eastward winds

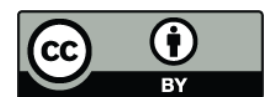

Correspondence to: C. Anderson (c.anderson@latrobe.edu.au) in the afternoon/evening generally continued until around local midnight, when winds turned equatorward. Geographic meridional and zonal winds in the afternoon were approximately $50 \mathrm{~ms}^{-1}$ weaker than expected from HWM93, as was the transition to equatorward flow around midnight. There was also a negligible geographic zonal component to the post-midnight wind where HWM93 predicted strong westward flow. Average temperatures between 19:00 and 04:00 local solar time were around $60 \mathrm{~K}$ higher than predicted by NRLMSISE-00.

Keywords. Atmospheric composition and structure (Airglow and aurora) - Meteorology and atmospheric dynamics (Thermospheric dynamics; Instruments and techniques)

\section{Introduction}

Large-scale thermospheric F-region winds are driven predominantly by the pressure gradient which results from solar heating on the dayside, and momentum transfer from ions convecting in the $\boldsymbol{E} \times \boldsymbol{B}$ direction due to electric fields mapped down from the magnetosphere. These driving forces tend to reinforce each other over the poles, forcing the neutral wind in the antisunward direction, while at auroral latitudes the return (sunward) flow of ions tries to set up a two-cell circulation of the neutral wind (Thayer and Killeen, 1993).

Recently, evidence has been growing that structures exist in the thermospheric wind field at spatial scales of several hundred kilometers or less (Conde and Smith, 1998; Aruliah and Griffin, 2001; Aruliah et al., 2004). Ongoing development of all-sky imaging Fabry-Perot spectrometers (Rees and Greenaway, 1983; Rees et al., 1984; Batten et al., 1988; Sekar et al., 1993; Biondi et al., 1995; Griffin et al., 2008) has resulted in instruments capable of recording independent spectra from many tens of locations across the sky simultaneously, making it possible to investigate this structure directly.

Published by Copernicus Publications on behalf of the European Geosciences Union. 


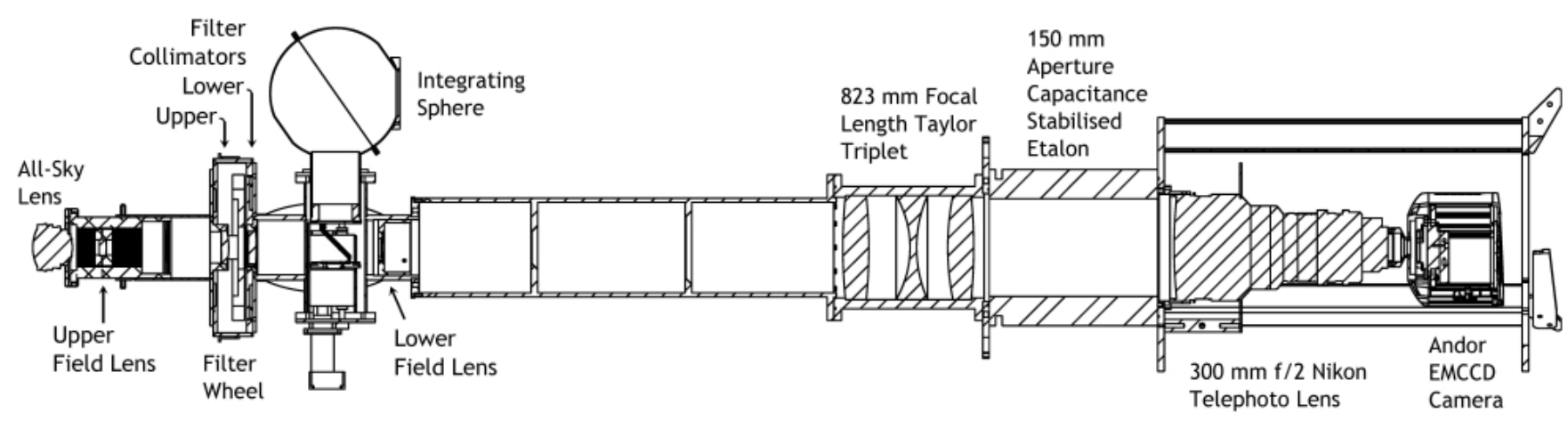

Fig. 1. Schematic of the Mawson all-sky imaging Fabry-Perot spectrometer.

Table 1. Instrument specifications.

\begin{tabular}{lr}
\hline Etalon & \\
\hline Nominal gap & $25 \mathrm{~mm}$ \\
Aperture & $150 \mathrm{~mm}$ \\
Max light incidence angle & $0.93^{\circ}$ \\
Reflective finesse & 13.5 \\
\hline Lens & Focal length $(\mathrm{mm})$ \\
\hline All-sky lens & 8 \\
Upper field lens & 50 \\
Filter upper collimator & 250 \\
Filter lower collimator & 300 \\
Lower field lens & 250 \\
\hline
\end{tabular}

F-region winds and temperatures have been observed previously from Mawson station, Antarctica, using a singleetalon, separation scanned, non-imaging ("pinhole scanner") Fabry-Perot spectrometer (Wardill et al., 1987; Conde and Dyson, 1995a,b; Innis et al., 1996; Greet et al., 1999). The $630.0 \mathrm{~nm}$ line of atomic oxygen was observed, yielding estimates of conditions in the auroral F-region at a typical height of approximately $240 \mathrm{~km}$. This instrument used a periscope to observe patches of the sky subtending around $1^{\circ}$ fullangle. The typical observing sequence directed the periscope at $60^{\circ}$ zenith angle in each of the four cardinal (NSEW) directions, and also in the zenith. A more advanced (higherthroughput), narrow-field, single-etalon imaging instrument is currently in operation at Davis station $\left(68^{\circ} 35^{\prime} \mathrm{S}, 77^{\circ} 58^{\prime} \mathrm{E}\right)$, some $635 \mathrm{~km}$ east of Mawson (Davies et al., 2006). Measurements of Doppler-shift from these periscope-equipped instruments are typically averaged in the east-west and north-south directions to generate zonal and meridional components of the overhead wind field. The wind vectors thus derived are estimates of the "background" wind direction over a large spatial extent.

A new all-sky imaging Fabry-Perot spectrometer was installed at Mawson station during the 2006/2007 summer, and began useful operation in March 2007. During the 2007 observing season (March-October) the instrument observed the $630.0 \mathrm{~nm}$ airglow line, and the data were used to derive Fregion winds and temperatures. These data are presented.

\section{Instrument description}

The instrument used to obtain the data presented here is an all-sky imaging, separation-scanned Fabry-Perot spectrometer, of the type described by Conde and Smith (1997). A schematic is shown in Fig. 1. It utilises an all-sky lens to pass an approximately $75^{\circ}$ half-angle field-of-view of the sky through a large $(150 \mathrm{~mm})$ aperture, capacitance-stabilised Fabry-Perot etalon. The gap between the etalon plates is piezoelectrically scannable over approximately 2.5 orders of interference at $\lambda 630.0 \mathrm{~nm}$, about a nominal gap of $25 \mathrm{~mm}$. Table 1 lists the key etalon properties, along with the focal lengths of lenses comprising the fore-optics. A six-position filter-wheel within the fore-optics allows for selection of observing wavelength. The wheel currently carries three interference filters, two for wavelengths of $630.0 \mathrm{~nm}$ and one for $557.7 \mathrm{~nm}$. Light from the etalon is angularly modulated by approximately 8 orders of interference fringes. A very fast, $300 \mathrm{~mm}$ F/2 Nikon lens images these fringes onto an Andor "Ixon" electron-multiplying camera. The camera is thermoelectrically cooled to $-80^{\circ} \mathrm{C}$ and achieves greater than $90 \%$ quantum efficiency throughout most of the visible spectrum, with very small read noise.

Calibration light from up to four different sources can be introduced directly beneath the filter-wheel via an integrating sphere. Two calibration sources were available during 2007, at wavelengths of $632.8 \mathrm{~nm}$ and $543.5 \mathrm{~nm}$. Selection of the calibration source is made possible by a servo-driven shutter mechanism, controlled in software. A second softwarecontrolled servo drives a small mirror used to switch the viewing direction between the sky and the calibration sphere.

The fringe-modulated image of the sky recorded by the camera is subdivided by the analysis software into a number 
of "zones" for the purpose of spectral accumulation, with each zone producing an individual spectrum, as shown in Fig. 2 (lower panel). Spectra are recorded by repeatedly varying (i.e., scanning) the etalon plate separation over one order of interference in $\mathrm{N}$ discrete steps; a single image (frame) is obtained from the camera at each step. For each image, signal "counts" from each pixel are appropriately summed to produce spectra. That is, the software first determines to which zone a pixel belongs, and then determines which spectral channel that pixel maps to at that point in the scan. As described by Conde and Smith (1998), the latter determination is based on both the position of the pixel and on the current spacing of the plates. A scan over one interference order thus produces $\mathrm{N}$ individual camera frames, with a typical integration time for each frame being $0.1 \mathrm{~s}$. $\mathrm{N}$ is configurable; it was set to 128 for the observations reported here. The total number of $\mathrm{N}$-frame scans that must be summed to produce a spectrum of adequate signal-to-noise ratio in each zone depends of course on the prevailing emission brightness. In operation, the signal-to-noise ratio of the accumulating spectra is dynamically monitored by the control software. The accumulation is continued either until the median signal-to-noise level across all zones reaches a predetermined value, or until a set maximum time elapses. This process of co-adding spectra over multiple scans comprises what we refer to as one "exposure". For the observations described here the overall integration times per exposure varied from approximately 2 min during active aurora to as much as 15 min during very quiet conditions.

\section{Data analysis}

Geophysical parameters are derived by fitting to each recorded spectrum a model (Gaussian) emission spectrum convolved with a previously measured instrument function, using the method described by Conde (2001). In applying this algorithm to passive Fabry-Perot spectra, aerosol backscatter is excluded from the model spectrum, and returned temperatures are appropriate to passively acquired spectra (in contrast to lidar backscatter spectra, which are Doppler broadened by twice the amount of passive spectra). Estimates of peak wavelength, Doppler temperature, emission and background intensity are obtained from these fits. Calibration spectra are acquired by observing light from a frequency-stabilised He-Ne laser $(\lambda 632.8 \mathrm{~nm})$. The frequency with which these spectra are acquired is configurable; for the data presented here a single calibration exposure was recorded after every five airglow exposures. Calibration spectra are used to measure the instrument function, while the peak wavelengths returned from fits to these spectra are used to correct derived winds for instrumental drift, which rarely exceeds an overall excursion of around $15 \mathrm{~ms}^{-1}$ during the night. The frequency drift of the laser is reported by the manufacturer (for a similar laser in use at Davis sta-

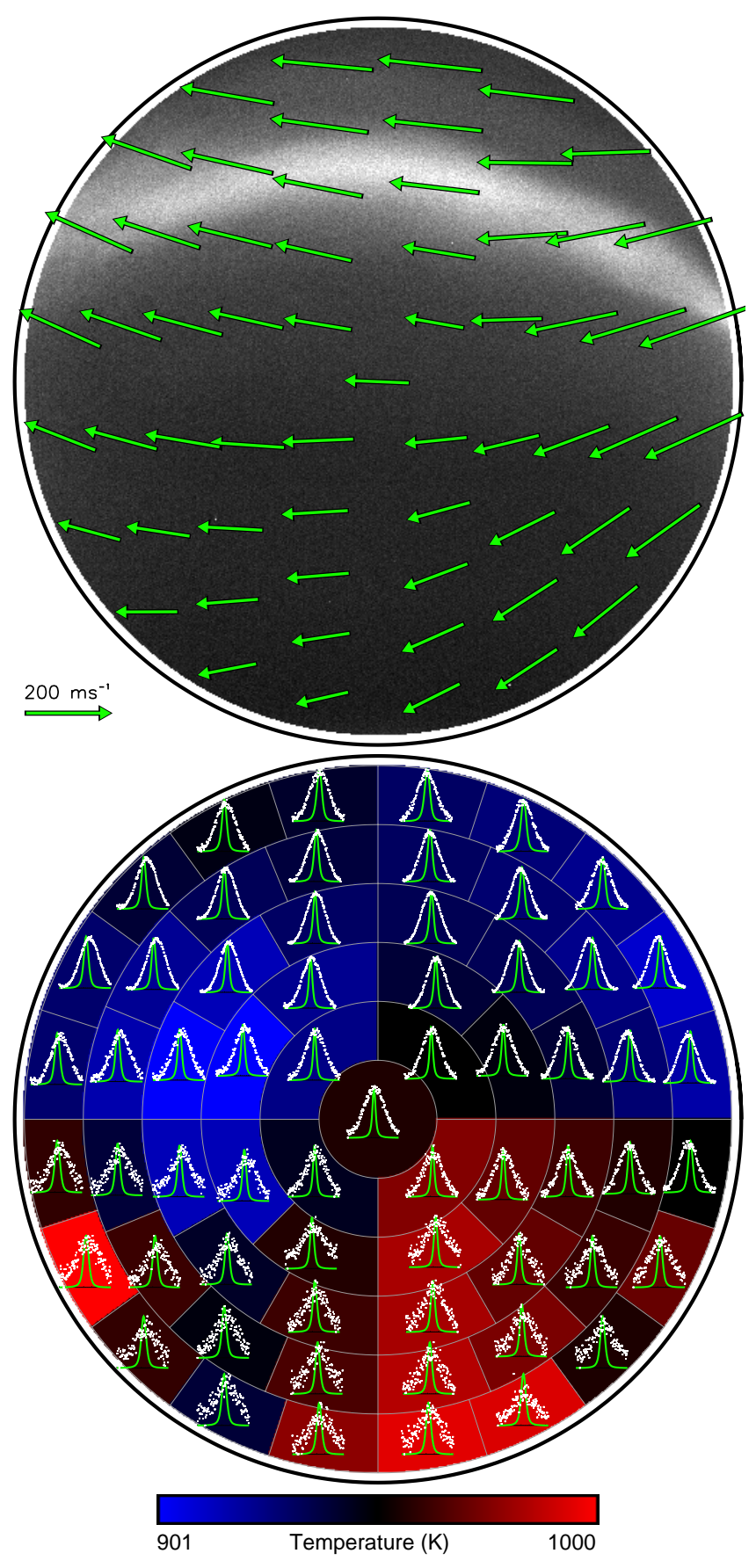

Fig. 2. Top: Fitted wind vectors (offset-corrected) superimposed over the corresponding all-sky image. Bottom: Zone map showing recorded spectra (white) and instrument profiles (green) for the same exposure. The temperatures estimated from these sky profiles are shown as blue/red hues (with the range indicated by the scale bar below the figure). This data was recorded on 23 May 2007, at 17:07 local solar time, with an exposure time of $150 \mathrm{~s}$. Magnetic north is at the top of these images, east is to the right. 
tion) to be $\pm 0.3 \mathrm{MHz}$ in one hour. The corresponding wavelength drift is approximately $5 \times 10^{-5}$ of the etalon free spectral range, which is negligible.

For the data presented here the field-of-view was divided into 61 zones. At $240 \mathrm{~km}$ altitude, where the peak of the $630.0 \mathrm{~nm}$ airglow emission occurs, the radial field-of-view of the instrument is approximately $740 \mathrm{~km}$. The horizontal (radial) extent of a zone projected onto the sky at this altitude depends on radial distance from the center of the field-ofview, and varies here from approximately $55 \mathrm{~km}$ for zones in the first annulus out from the center, to $320 \mathrm{~km}$ for the outermost annulus. A zero-velocity reference is obtained by assuming an average vertical wind speed of $0 \mathrm{~ms}^{-1}$ throughout the night. Although this assumption is likely not valid for shorter periods of observation, the errors introduced by this assumption are at most around $20 \mathrm{~ms}^{-1}$ (Aruliah and Rees, 1995), and likely much less. Vertical wind is recorded in the central zone, and line-of-sight winds are calculated by comparing peak wavelength time-series for each zone to the zero-velocity reference.

Having reduced the spectral data to time series of zonedependent peak-position, temperature, background and intensity, a second level of analysis is used to fit a horizontal vector wind field to the subset of line-of-sight peak-positions which comprise each exposure. See Conde and Smith (1998) for a detailed discussion of this vector fitting method. The vector fit describes the two-dimensional wind field as a superposition of uniform wind terms (mean meridional and zonal winds, calculated separately in each annulus) and the gradients of these winds in each of the meridional and zonal directions. The latter can only be determined in annuli containing $\geq 6$ zones, and are averaged over all such annuli to produce a single all-sky mean value for each gradient term. The final vector components for each zone are then obtained from the uniform wind terms (calculated separately within each annulus) and the gradient terms averaged over the fieldof-view. The central zone, which does not contribute to the vector fit, is used to hold the median values of each wind component returned by the fitting algorithm. When plotted, the central zone thus shows the average wind direction and magnitude across all zones for each exposure. Note that only the horizontal wind field is fitted in this way; temperatures and intensities are returned by the spectral fitting algorithm independently for each zone.

For every channel in a scan the camera records a monochromatic, fringe-modulated image of the sky at the wavelength of observation. The superposition of all such images in a single scan allows the instrument to build up a normal $512 \times 512$ pixel sky image after each scan is completed. The final sky image obtained from an overall exposure (comprised of multiple scans) is the sum of all these single-scan sky images. An example all-sky image, with the corresponding fitted vector wind field superimposed on it, is shown in Fig. 2 (upper panel), along with the recorded sky spectra, instrument profiles and temperature estimates (lower panel).
Importantly, these images are obtained at the same wavelength as the corresponding wind and temperature estimates, over identical fields of view and identical integration periods.

The analysis to this point is useful for examining the dayto-day variability of the thermospheric wind and temperature field. However in order to investigate the average behavior of these winds and temperatures, also known as a superposed epoch analysis, a third level of analysis is required. For each exposure, median values of the fitted meridional and zonal wind (i.e. the average wind components stored in the central zone) and temperature are calculated to produce a single all-sky average value for these parameters in each exposure. Corresponding to these observed (average) values, model estimates of wind and temperature are produced for each exposure using HWM93 and NRLMSISE-00 respectively, as described in Sect. 3.2. A single estimate of zonal and meridional wind, plus temperature, is produced for each exposure, using the geomagnetic and solar conditions prevailing at the time the exposure was made. Exposures are then sorted by geomagnetic and solar activity (parameterised here by the Ap and F10.7 indices respectively) and cloud level. The all-sky median values from those exposures which meet the selection criteria (in this case ranges of activity and cloud levels) are then sorted into time bins of configurable width. The median of each time bin then represents the average value of a certain parameter (including both observed and modelled winds and temperatures) occurring within a particular time interval and under the given geomagnetic and solar conditions.

\subsection{Offset correction}

Cloud appearing in the instrument's field-of-view can affect line-of-sight velocity estimates, as cloud can scatter the optical signal both into and out of the line-of-sight direction. If the unscattered component of the brightness in a particular direction is low compared to much brighter regions in other parts of the sky, the net signal seen when viewing the cloud may include a significant or even dominant contribution from directions other than the original line-of-sight. This essentially produces an intensity-weighted spatial average over a region of the sky that extends beyond the original viewing zone. Depending on the angular distribution of emission brightness and Doppler-shift across the sky, such scattering can shift the apparent wavelength seen when viewing cloud, relative to that which would be seen when viewing the sky directly. In the case of very heavy cloud, the averaging occurs across the whole sky, and is so complete that the line-of-sight velocity measured by all zones will approach zero.

However, exposures recorded by the Mawson instrument during such periods of very heavy cloud (covering the entire field-of-view) show non-zero line-of-sight velocities whose magnitude varies with azimuth around the detector, consistent with a constant, non-zero offset aligned along the detector's top-bottom axis. Selecting this axis to represent the 

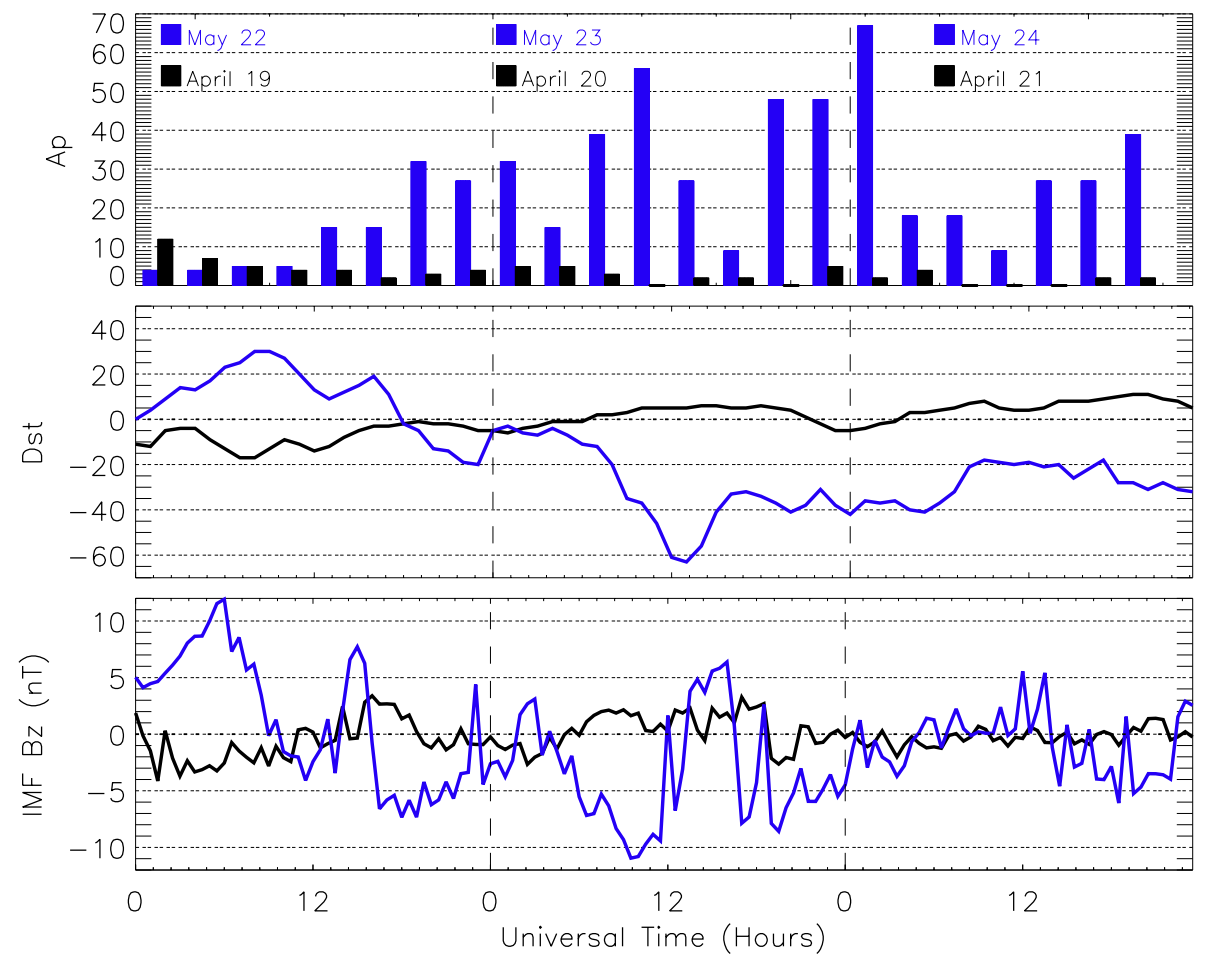

Fig. 3. Geomagnetic conditions for the 3-day periods centered on nights A and B. Night A (20 April) is shown in black, night B (23 May) in blue.

magnetic meridional direction (with positive directed from the bottom to the top of the detector), the effect of this offset is to add a magnetic meridional component of approximately $-45 \mathrm{~ms}^{-1}$ (i.e. magnetically southward) to the fitted horizontal wind field. In correcting this offset, an average line-of-sight wind velocity was obtained for each zone during 12 days which had very heavy cloud cover (time averaging over 12 days should further suppress any angular variation of Doppler-shift over the actual sky). These line-of-sight velocities were then subtracted from each exposure before fitting the vector wind field. This offset is clearly present and reproducible across multiple calibrations. While the exact mechanism responsible for it is currently unclear, we have indications from both this instrument and from a similar one in Alaska that it is related to the way charge was read out from the camera's CCD chip. Results from a (currently unpublished) study into vertical winds using both the Mawson and Davis spectrometers have confirmed the need for this offset correction.

\subsection{Empirical and numerical models}

Three atmospheric models were used for comparison in this study. The 1993 Horizontal Wind Model (HWM93) (Hedin et al., 1988, 1991) is an empirical model of the horizontal neutral wind in the upper atmosphere. Inputs include alti- tude, latitude, longitude, time, solar radio flux (F10.7) and magnetic activity (Ap) index. NRLMSISE-00 (Picone et al., 2002 ) is an empirical model which outputs neutral temperature and density from ground to thermospheric heights. Inputs are almost identical to HWM93. The Coupled Thermosphere/Ionosphere Plasmasphere (CTIP v1.0) model (FullerRowell et al., 1996; Millward et al., 1996) solves the timedependent, coupled equations of momentum, energy and continuity to calculate the temperature, density and velocity of the neutral atmosphere, as well as ion temperatures and densities over selected height ranges. Inputs to this model include fixed or time-dependent values of Hemispheric Power, Hemispheric Power Index, solar radio flux (F10.7) and solar wind parameters.

\section{Results}

Winds and temperatures derived from observations of the $630.0 \mathrm{~nm}$ airglow line of atomic oxygen are presented in two parts. First, two examples of individual nights of observation are presented, to highlight the day-to-day variability and the contrast between geomagnetically quiet and active conditions. Comparisons are made with the models described in Sect. 3.2, where the inputs to all three models were constant (i.e. time-independent), and represented the mean 

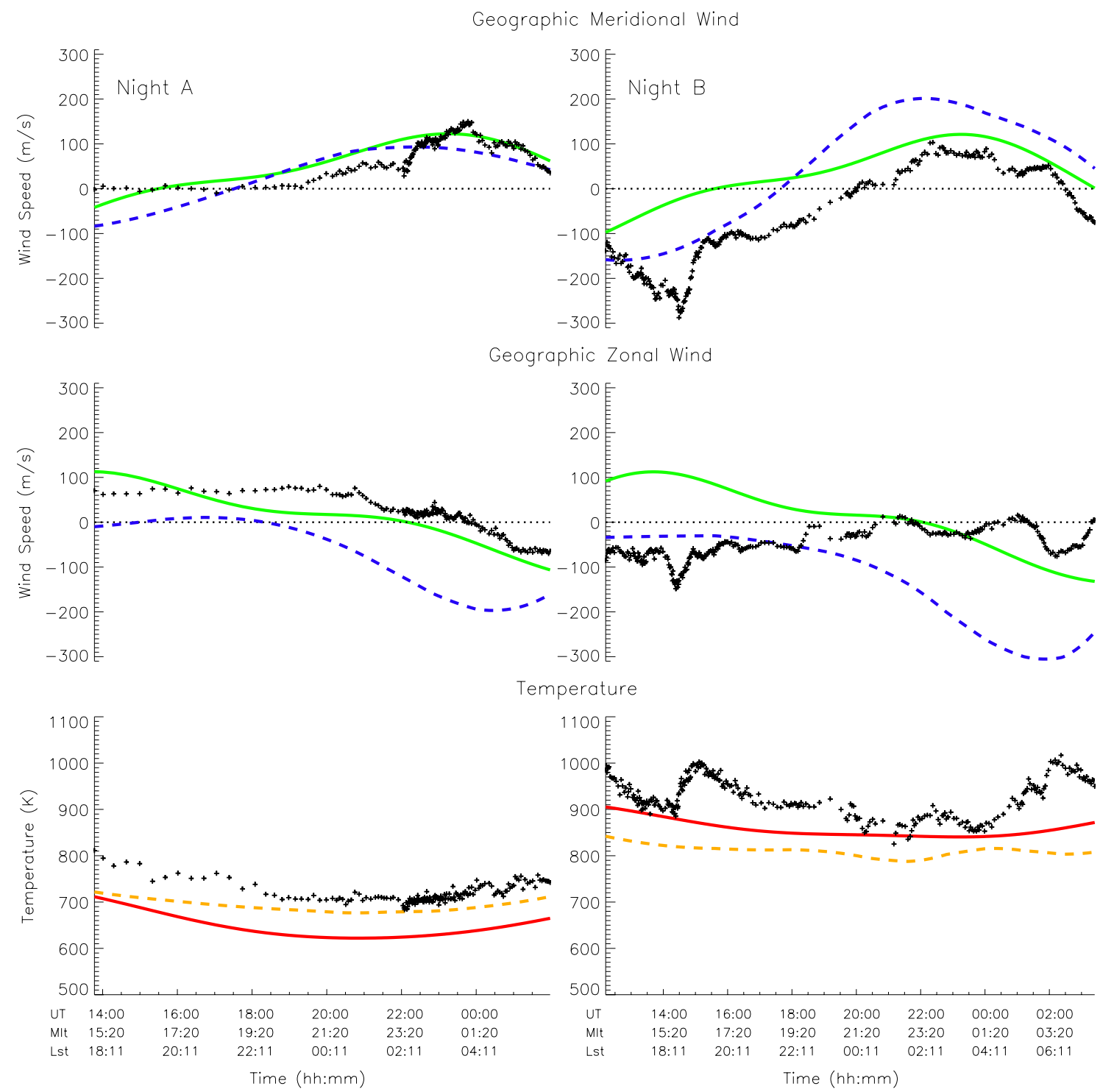

mperature

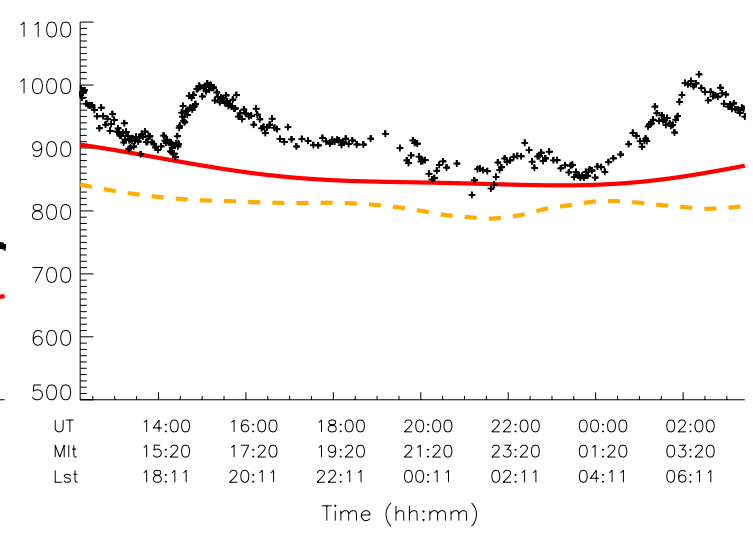

Fig. 4. Winds and temperatures observed on nights A and B. Typical uncertainty in observed wind speed and temperature is around $10 \mathrm{~ms}^{-1}$ and $45 \mathrm{~K}$, respectively. HWM93 (solid green curve) and CTIP (dashed blue curve) predicted winds are shown, along with temperatures output by CTIP (dashed orange curve) and NRLMSISE-00 (solid red curve). Inputs to models were constant over the observing periods.

geomagnetic and solar conditions prevailing during each observing period.

Secondly, average time-series of wind components and temperature are shown, for observations made under all (cloud-free) conditions, and comparisons with HWM93 and NRLMSISE-00 are made. While geomagnetic activity was quite variable (though low) for this data set, solar activity remained consistently low during these solar minimum conditions.

\subsection{Daily variability}

Data from two nights of observation, 20 April and 23 May 2007, will be discussed in detail. These nights will be referred to as nights $\mathrm{A}$ and $\mathrm{B}$, respectively. Geomagnetic conditions and the $B_{z}$ component of the IMF for the three days centered on each of these nights are shown in Fig. 3. Night A was a time of very low activity. Ap reached a maximum of 12 the previous day, and for the rest of the 3 day period was generally less than 5. $D_{s t}$ and IMF $B_{z}$ also show minimal disturbance. Night $B$ was conversely a period of relatively active geomagnetic conditions, with a rising trend in Ap (beginning around 12:00 UT the previous day) accompanied by strongly negative $D_{s t}$ and southward IMF. 

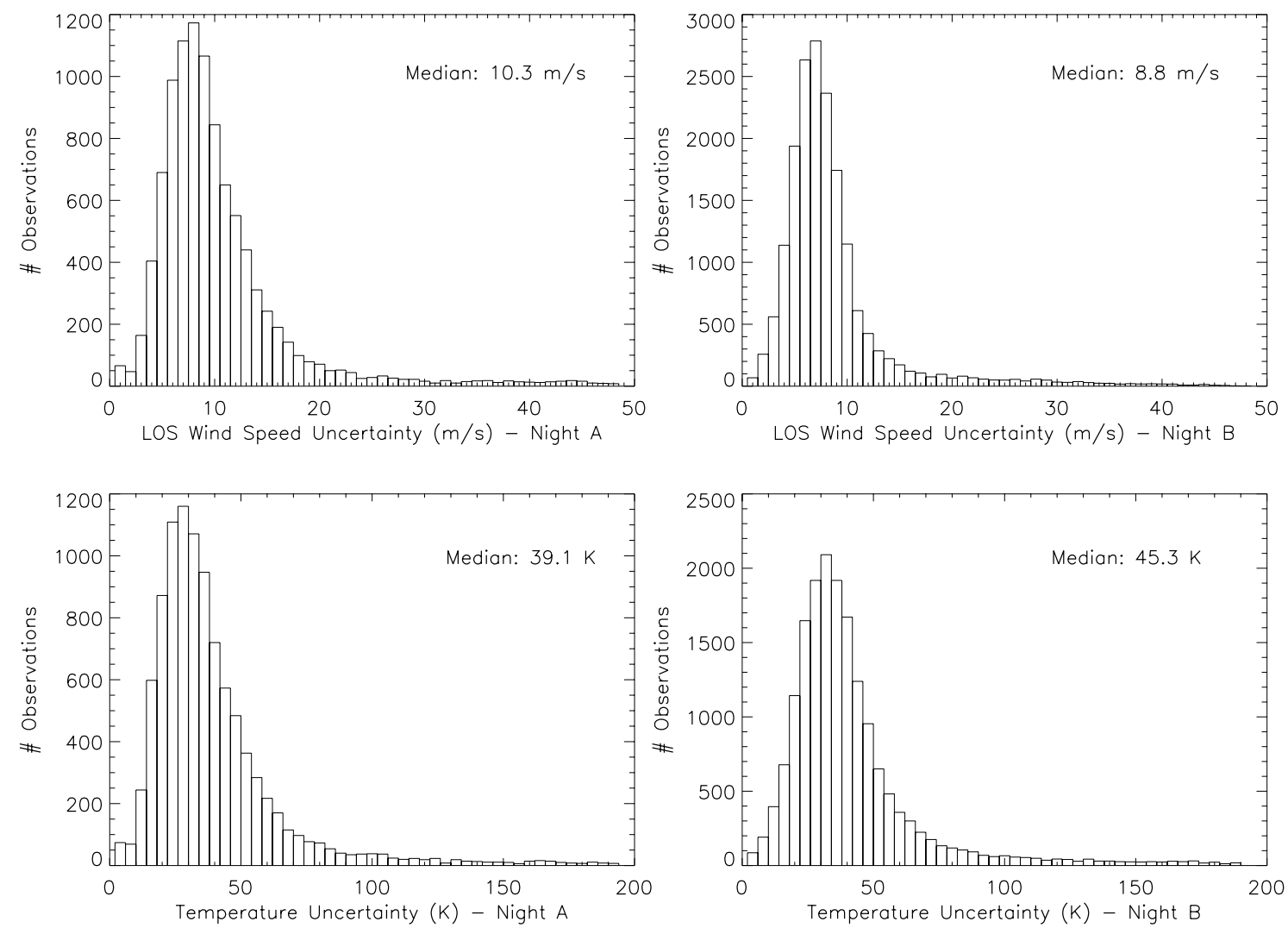

Fig. 5. Histograms of line-of-sight wind and temperature uncertainties for nights A and B, across all zones.

Figure 4 shows the observed winds and temperatures for nights $\mathrm{A}$ and B. Geographic meridional and zonal components of the fitted wind field, as well as temperature, are median values over the entire field of view. Winds output by HWM93 (solid green line) and CTIP (dashed blue line), as well as temperatures from CTIP (dashed orange line) and NRLMSISE-00 (solid red line) are shown. As mentioned previously, inputs for all three models were constant throughout the observing periods, and were representative of the mean prevailing conditions. The effect of increased signal level on exposure times can be seen in the decreased temporal spacing of data points on night $\mathrm{B}$ compared to night $\mathrm{A}$.

Figure 5 shows histograms of the 1- $\sigma$ line-of-sight wind and temperature uncertainties for each night, across all zones. These are the statistical uncertainties in the absolute values of the line-of-sight winds and temperatures returned by the spectral fitting algorithm, and give a good indication of instrumental performance. Typical uncertainty in fitted line-of-sight wind speed is around $10 \mathrm{~ms}^{-1}$, while temperature uncertainty is usually less than $45 \mathrm{~K}$.

Night A was a good example of what is often seen at Mawson. Wind flow was (geographically) antisunward in the evening, and remained eastward for most of the night. The wind became predominantly northward around $2 \mathrm{~h}$ after lo- cal midnight. Meridional winds were reasonably well modelled by both HWM93 and CTIP, however CTIP predicted zonal winds which were offset from those observed by 70$180 \mathrm{~ms}^{-1}$ westward. Temperatures were between $50-100 \mathrm{~K}$ higher than those predicted by NRLMSISE-00, but followed the same trend, decreasing smoothly from $800 \mathrm{~K}$ at the start of the night to a minimum of $680 \mathrm{~K}$ around local midnight, before once again increasing to approximately $750 \mathrm{~K}$ at the end of the night (6am local solar time). CTIP temperatures were in much closer agreement with those observed, particularly towards the end of the night. Discrepancies between observed and modelled temperatures are unlikely to be due to any instrumental effects since the instrument function is much narrower than the emission spectrum.

Winds on night B were poorly modelled by both HWM93 and CTIP. Afternoon/evening sector winds showed strong (up to $300 \mathrm{~ms}^{-1}$ ) flow southward across the geographic pole. At Mawson this translates to a wind aligned strongly with geomagnetic west, following the plasma convection. Equatorward flow was seen around $2 \mathrm{~h}$ after local midnight, after which time the observatory passed beneath a region of weak, variable zonal wind. Meridional winds predicted by HWM93 and CTIP for this night both had the correct morphology with time, however both were offset by around $70-100 \mathrm{~ms}^{-1}$ 


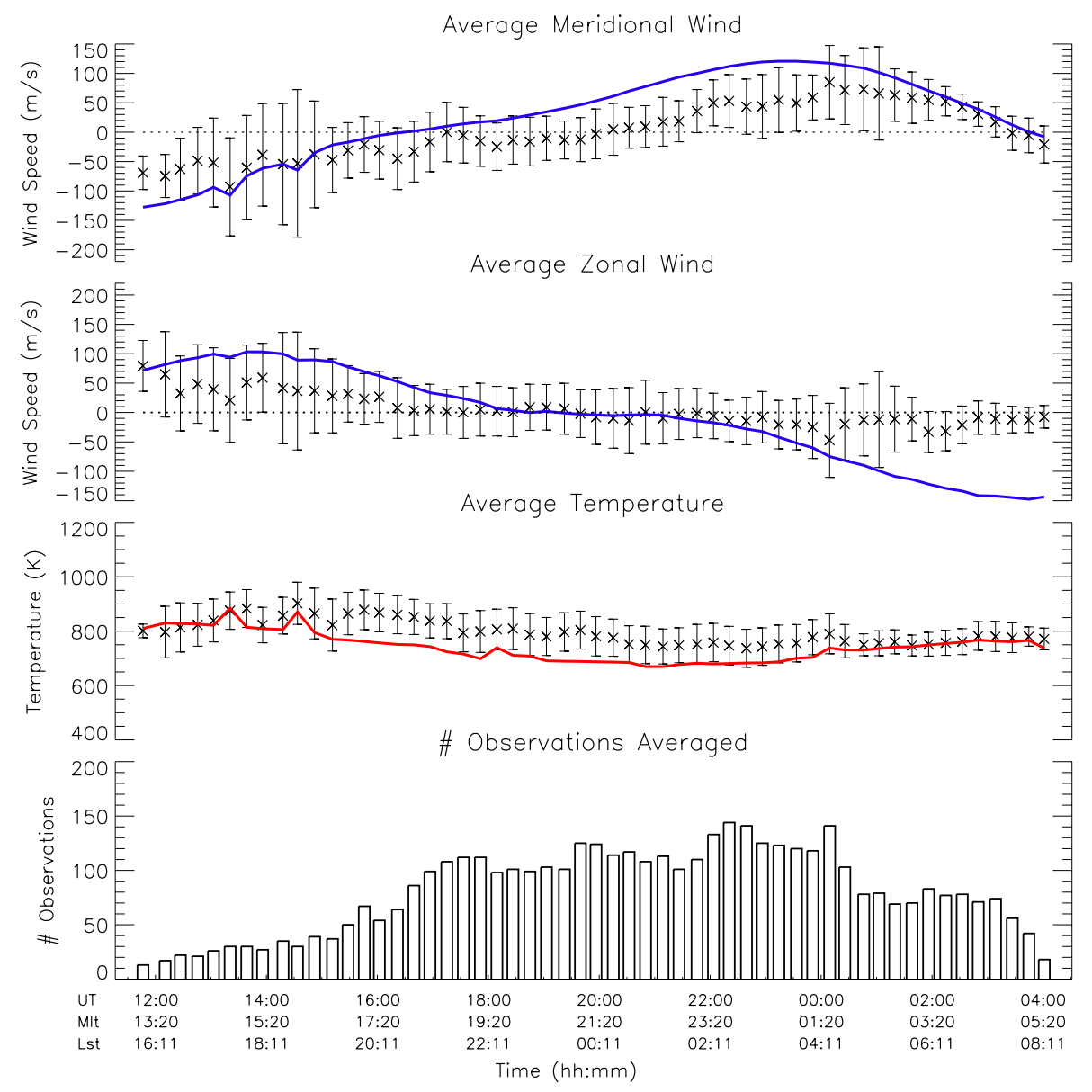

Fig. 6. Average winds and temperatures observed under all geomagnetic conditions. Error-bars indicate the 1- $\sigma$ uncertainties of the observed distributions within each time bin. Solid blue and red lines show model output (HWM93 for winds, NRLMSISE-00 for temperatures) for the averaged geomagnetic conditions.

northward, and CTIP predicted a larger amplitude to the nightly variation than was observed. Both models predicted a similar trend in the zonal wind (with CTIP offset from HWM93 by $100-200 \mathrm{~ms}^{-1}$ westward), which was in poor agreement with observation.

Median temperature on this night was around $200 \mathrm{~K}$ higher than night $\mathrm{A}$, and showed much more variability during the night. These fluctuations were not reproduced by either NRLMSISE-00 or CTIP, however temperatures predicted by NRLMSISE-00 better matched our data on this night than did those predicted by CTIP. Temperature fluctuations as large as $100 \mathrm{~K}$ were seen to occur over a relatively short time (approximately $45 \mathrm{~min}$ ). These fluctuations were likely due to a combination of actual heating (both Joule and particle heating) and a changing height profile of the $630.0 \mathrm{~nm}$ emission in response to variations in the energy spectrum of the auroral precipitation. The minimum (median) temperature of $830 \mathrm{~K}$ occurred around $1 \mathrm{a} . \mathrm{m}$. local solar time.

\subsection{Average daily behavior}

Figure 6 shows the results of the superposed epoch analysis outlined in Sect. 3, where the epoch is in this case $24 \mathrm{~h}$ of local time. For this analysis all exposures recorded when cloud levels were $\leq \frac{3}{8}$ (a total of 80 nights) were binned into approximately $20 \mathrm{~min}$ time intervals, and the median value within each time bin obtained. Error bars show the $1-\sigma$ widths of the observed distributions within each time bin. Model winds and temperatures output by HWM93 and NRLMSISE-00 are shown as solid blue and red lines respectively, while the bottom panel shows the number of exposures contributing to each time bin. As described in Sect. 3, model values are calculated for every exposure in the data set, and averaged in the same way as observed winds and temperatures. Each time bin then contains, along with the average of the observed wind components and temperatures, the average of the returned model estimates corresponding to each of these parameters, for the geomagnetic and solar activity 
$\stackrel{200 \mathrm{~m} / \mathrm{s}}{\longrightarrow}$

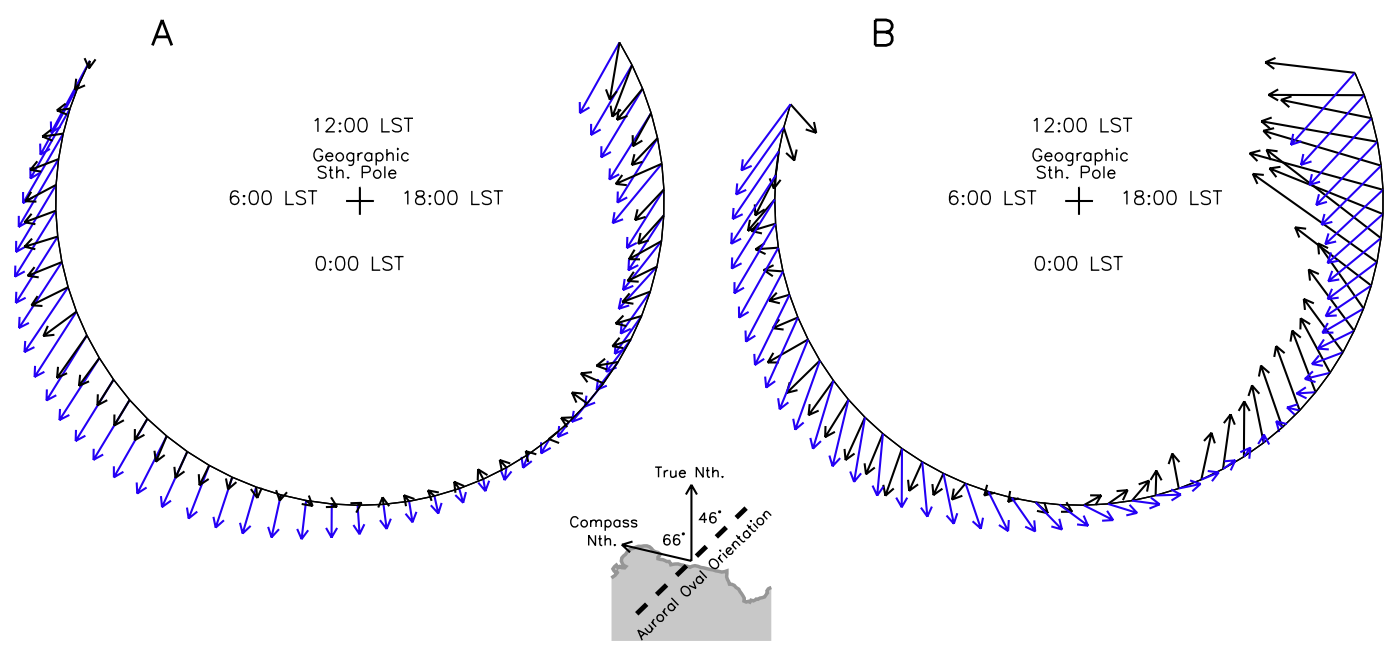

Fig. 7. (A) Dial-type representation of Fig. 6, showing average winds observed under all geomagnetic and solar conditions, which for this data set is representative of a quiet night at solar minimum. (B) Dial-type representation of Night B (active conditions), shown for comparison with the average, geomagnetically quiet night shown in (A). In both plots, black arrows show observed (averages in plot A) wind direction, blue arrows show HWM93 model winds, in geographic coordinates. Local solar time increases clockwise around the dial. The sun is to the top of the figure. The small map underneath shows the geographic north and compass north directions, relative to the Mawson coastline, as well as the orientation of the auroral oval relative to these directions.

prevailing at the time those observations were made. Alternative model averaging schemes were tested, and found to have only minor impact on the resulting average model timeseries. It is for example possible to use the mean value of Ap and F10.7 within each time bin as input to the models, or to use only a single March-October average for each of these inputs, over the entire epoch. We believe the current scheme best represents the average model results, despite the fact that some small discontinuities remain when these results are viewed as an average time-series.

The data set from which these averages were calculated contained very few geomagnetically active days. Of those observations which were made when cloud was $\leq \frac{3}{8}, 84 \%$ were made during periods when Ap was $\leq 20$. Solar activity was also very low, as F10.7 showed minimal variation around a median of $71 \times 10^{-22} \mathrm{~J} \mathrm{~s}^{-1} \mathrm{~m}^{-2} \mathrm{~Hz}^{-1}$. Thus the data set is heavily biased towards a geomagnetically quiet day under solar minimum conditions. The contribution to any given time bin from observations made under active geomagnetic conditions has been shown to be minimal by comparing Fig. 6 with one produced by observations which were limited to those made when Ap was $\leq 10$ (not shown). For the majority of the night, the difference is less than $20 \mathrm{~ms}^{-1}$ for wind speeds and $30 \mathrm{~K}$ for temperatures. These values are small compared with the 1- $\sigma$ widths within each time bin, and would have very little impact on the following discussion.

Figure 7a presents the data of Fig. 6 in a dial-type plot. Observations have been interpolated to show 50 wind vec- tors between the earliest and latest times for which there is data available. Here wind vectors are plotted from the perspective of an observer looking down on the geographic south pole (shown in the figure as a cross), from a point in space above the pole. The geographic southward direction therefore points radially inward at all times, while the eastward direction is clockwise around the dial. Local solar time (approximately $4 \mathrm{~h}$ ahead of universal time at Mawson) increases clockwise around the dial, and the sun is to the top of the figure. Wind vectors predicted by HWM93 are shown in blue.

The general trend seen above Mawson was for antisunward (geographic eastward) winds in the afternoon/evening sector. From approximately 21:00 local solar time wind speeds were much reduced, and remained low until the transition to geographic northward flow occurred approximately $2 \mathrm{~h}$ after local midnight. After this transition, geographic meridional wind speeds were often relatively slow, around $50-80 \mathrm{~ms}^{-1}$. Agreement between observed and modelled winds was reasonable except for the lack of a strong westward zonal wind in the morning sector expected by HWM93. Also the transition to equatorward flow around this time often exhibited slower meridional wind speeds than was predicted by the model. It should be noted that a large amount of data contributing to the empirical HWM93 were recorded during solar maximum conditions, which may account for some of the discrepancies between observed and modelled winds, particularly with regard to wind speed. 
The average winds presented here are similar to those found in comparable studies of thermospheric winds above Mawson, see for example Conde and Dyson (1995a). However, in this earlier study, strong (100-200 $\left.\mathrm{ms}^{-1}\right)$ average winds were observed flowing geomagnetically equatorward after 02:50 local solar time (magnetic midnight), whereas the average data presented here show very weak flow in this time sector, directed magnetically eastward. These authors also observed predominantly magnetic zonal flow between 21:50 and 00:50 local solar time, whereas the present study shows a stronger magnetic poleward component in this period. The study cited was carried out during the downward phase of solar cycle 22, under conditions of greater solar activity, which may account for some of these differences.

Since the average winds shown in Fig. 7a are representative of a geomagnetically quiet night, Fig. $7 \mathrm{~b}$ is given to illustrate the effect that increased geomagnetic disturbance has on observed winds. This dial-plot is in the same format as Fig. 7a, and shows the wind vectors observed on night B (Sect. 4.1), during which time levels of geomagnetic activity were relatively high (see Fig. 3).

The most noticeable feature of the wind on this night is the strong geographic poleward component in the afternoon and evening, which was present at reduced magnitude until local midnight. Since geomagnetic north at Mawson is approximately $66^{\circ}$ west of true north, this poleward wind is directed approximately sunward in a geomagnetic coordinate system, following the plasma convection. Wind speeds are much stronger throughout this period than predicted by HWM93.

Average temperatures show better agreement with NRLMSISE-00 at the beginning and end of the observation period. For the $9 \mathrm{~h}$ or so between 15:00 and 24:00 UT temperatures were often around $60 \mathrm{~K}$ higher than predicted by the model, similar to the quiet night (night A) presented in Sect. 4.1. Since NRLMSISE-00 relies on experimental data input, this disagreement between the average observed temperatures and model temperatures is possibly due to a lack of data coverage in the model above Mawson at these times, and under quiet geomagnetic conditions. Agreement with the model generally improved with increasing geomagnetic activity. Mean temperature was approximately $790 \mathrm{~K}$.

\section{Conclusions}

Data from the first season of operation of the Mawson all-sky imaging Fabry-Perot spectrometer have been presented. Initial analysis has focused on the behaviour of winds and temperatures across the entire field-of-view of the instrument. For geomagnetically quiet nights the largescale winds followed reasonably well the trends predicted by HWM93 and CTIP, although CTIP predicted zonal winds which were offset by $70-180 \mathrm{~ms}^{-1}$ westward from those observed. Temperatures were well modelled by CTIP, but were often higher than those predicted by NRLMSISE-00, by ap- proximately $100 \mathrm{~K}$. On nights of above average geomagnetic activity however the meridional and zonal components of the wind field can show much greater departures from modelled winds, and much greater variability over shorter time-scales. These departures are not surprising considering the highly variable nature of energy inputs to the auroral thermosphere (see for example Kosch et al., 2000). For the active night presented here, median temperatures were up to $200 \mathrm{~K}$ higher, and showed large (up to $100 \mathrm{~K}$ ) short-lived variations.

Average behavior was characterised by antisunward winds in the late afternoon which continued into the pre-midnight sector at a reduced magnitude, with northward flow occurring around $2 \mathrm{~h}$ after local midnight. Wind speeds were generally lower than expected, which may be attributable to deep solar minimum conditions as mentioned previously. Temperatures followed the trend expected by NRLMSISE-00, although they were higher by around $60 \mathrm{~K}$ during the middle of the observation period.

While initial analysis has focused on the large-scale daily and average behaviour of winds and temperatures, an imaging instrument such as the one employed here is ideally suited to the observation of small-scale structures. Figure 2 shows an example of such structure, in this case divergence in the wind field. This figure also shows an approximately $100 \mathrm{~K}$ temperature variation in the vicinity of an auroral arc, another feature which is commonly observed. This smallscale structure will be the focus of future study.

Acknowledgements. This research has been supported by the Australian Research Councils Discovery Project DP0557369 and by the Australian Antarctic Science Program. Logistic support for this work was provided by the Australian Antarctic Division. Many parts of the FPS used in this project were constructed at La Trobe University by Eric Huwald, Habib Rahman and Robert Polglase. The NRLMSISE-00 and HWM93 models are provided by the Community Coordinated Modeling Center (CCMC). The CTIP model was run through the CCMC's web interface, and help was provided by model developers Tim Fuller-Rowell and Mihail Codrescu. The authors wish to acknowledge the continuing support of the ANARE expeditioners at Mawson station, without which this work would not have been possible.

Topical Editor C. Jacobi thanks D. McEwen, T. Sakanoi, and R. Niciejewski for their help in evaluating this paper.

\section{References}

Aruliah, A. L. and Rees, D.: The trouble with thermospheric vertical winds: geomagnetic, seasonal and solar cycle dependence at high latitudes, J. Atmos. Terr. Phys., 57, 597-609, 1995.

Aruliah, A. L. and Griffin, E.: Evidence of meso-scale structure in the high-latitude thermosphere, Ann. Geophys., 19, 37-46, 2001, http://www.ann-geophys.net/19/37/2001/.

Aruliah, A. L., Griffin, E. M., McWhirter, I., Aylward, A. D., Ford, E. A. K., Charalambous, A., Kosch, M. J., Davis, C. J., and Howells, V. S. C.: First tristatic studies of meso-scale ion-neutral dynamics and energetics in the hight-latitude upper atmosphere us- 
ing collacted FPIs and EISCAT radar, Geophys. Res. Lett., 31, L03802, doi:10.1029/2003GL018469, 2004.

Batten, S., Rees, D., and Wade, D.: Observations of thermospheric neutral winds by the UCL Doppler imaging system at Kiruna in northern Scandinavia, J. Atmos. Terr. Phys., 50, 861-888, 1988.

Biondi, M. A., Sipler, D. P., Zipf, M. E., and Baumgardner, J. L.: All-sky Doppler interferometer for thermospheric dynamics studies, Appl. Optics, 34, 1646-1654, 1995.

Conde, M. and Dyson, P. L.: Thermospheric horizontal winds above Mawson, Antarctica, Adv. Space Res., 16, 41-52, 1995a.

Conde, M. and Dyson, P. L.: Thermospheric vertical winds above Mawson, Antarctica, J. Atmos. Terr. Phys., 57, 589-596, 1995 b.

Conde, M. and Smith, R. W.: 'Phase compensation' of a separation scanned, all-sky imaging Fabry-Perot spectrometer for auroral studies, Appl. Optics, 36, 5441-5450, 1997.

Conde, M. and Smith, R. W.: Spatial structure in the thermospheric horizontal wind above Poker Flat, Alaska, during solar minimum, J. Geophys. Res., 103(A5), 9449-9472, 1998.

Conde, M.: Analysis of Fabry-Perot spectra of lidar backscatter echoes, in: ANARE Reports, edited by: Morris, R. J. and Wilkinson, P. J., 146, pp. 91-114, Antarctic Division, Department of the Environment and Heritage, 2001.

Davies, T. P., Dyson, P. L., Anderson, C. E., and Conde, M. G.: A new Fabry-Perot spectrometer for thermospheric airglow observations above Davis station in Antarctica, Australian Institute of Physics (AIP) 17th Congress, Paper 40, Brisbane, Qld, Australia, 2006.

Fuller-Rowell, T. J., Rees, D., Quegan, S., Moffet, R. J., Codrescu, M. V., and Millward, G. H.: A coupled thermosphere-ionosphere model (CTIM), in: STEP Handbook on Ionospheric Models, edited by: Schunk, R. W., p. 217, Utah State University, 1996.

Greet, P. A., Conde, M. G., Dyson, P. L., Innis, J. L., Breed, A. M., and Murphy, D. J.: Thermospheric wind field over Mawson and Davis, Antarctica; simultaneous observations by two Fabry-Perot spectrometers of $\lambda 630 \mathrm{~nm}$ emission, J. Atmos. Solar-Terr. Phys., 61, 1025-1045, 1999.

Griffin, E. M., Aruliah, A. L., McWhirter, I., Yiu, H.-C. I., Charalambous, A., and McCrea, I.: Upper thermospheric neutral wind and temperature measurements from an extended spatial field, Ann. Geophys., 26, 2649-2655, 2008, http://www.ann-geophys.net/26/2649/2008/.

Hedin, A. E., Spencer, N. W., and Killeen, T. L.: Empirical Global Model of Upper Thermosphere Winds Based on Atmosphere and Dynamics Explorer Satellite Data, J. Geophys. Res., 93, 99599978, 1988.
Hedin, A. E., Biondi, M. A., Burnside, R. G., Hernandez, G., Johnson, M., Killeen, T. L., Mazaudier, C., Meriwether, J. W., Salah, J. E., Sica, R. J., Smith, R. W., Spencer, N. W., Wickwar, V. B., and Virdi, T. S.: Revised Global Model of Thermosphere Winds Using Satellite and Ground-Based Observations, J. Geophys. Res., 96, 7657-7688, 1991.

Innis, J. L., Greet, P. A., and Dyson, P. L.: Fabry-Perot spectrometer observations of the auroral oval/polar cap boundary above Mawson, Antarctica, J. Atmos. Terr. Phys., 58, 1973-1988, 1996.

Kosch, M. J., Ishii, M., Nozawa, S., Rees, D., Cierpka, K., Kohsiek, A., Schlegel, K., Fujii, R., Hagfors, T., Fuller-Rowell, T. J., and Lathuillere, C.: A Comparison of Thermospheric Winds and Temperatures from Fabry-Perot Interferometer and EISCAT Radar Measurements with Models, Adv. Space Res., 26, 979984, 2000.

Millward, G. H., Moffet, R. J., Quegan, S., and Fuller-Rowell, T. J.: A coupled thermoshere-ionosphere-plasmasphere model CTIP, in: STEP Handbook on Ionospheric Models, edited by: Schunk, R. W., p. 239, Utah State University, 1996.

Picone, J. M., Hedin, A. E., Drob, D. P., and Aikin, A. C.: NRLMSISE-00 empirical model of the atmosphere: Statistical comparisons and scientific issues, J. Geophys. Res., 107(A12), 1468, doi:10.1029/2002JA009430, 2002.

Rees, D. and Greenaway, A. H.: Doppler imaging system; an optical device for measuring vector winds. 1: General principles, Appl. Optics, 22, 1078-1083, 1983.

Rees, D., Greenaway, A. H., Gordon, R., McWhirter, I., Charleton, P. J., and Steen, A.: The Doppler imaging system: initial observations of the auroral thermosphere, Planet. Space Sci., 32, 273-285, 1984.

Sekar, R., Gurubaran, S., and Sridharan, R.: All sky imaging FabryPerot spectrometer for optical investigation of the upper atmosphere, Ind. J. Radio Space Phys., 22, 197-204, 1993.

Thayer, J. P. and Killeen, T. L.: A kinematic analysis of the highlatitude thermospheric neutral circulation pattern, J. Geophys. Res., 98, 11549-11565, 1993.

Wardill, P., Jones, N., and Jacka, F.: Dynamics of the Thermosphere over Mawson, Antarctica: I. Diurnal Variation and Geomagnetic Dependence, in: Anare Res. Notes, edited by: Burns, G. B. and Craven, M., 48, pp. 114-130, Antarctic Division, Department of Science, 1987. 